\title{
The research of improved ray casting algorithm on VTK
}

\author{
Hongtao Wei , Jin Li \\ School of Information Engineering, Wuhan University of Technology Wuhan 430070, China \\ 506991659@qq.com
}

Keywords: ray-casting, empty voxel skipping, multi-step length, visualization Toolkit.

\begin{abstract}
In the medical image visualization, ray-casting algorithm is a common algorithm. However, traditional ray-casting algorithm is not efficient for real-time volume rendering because of the excessive calculation and the high redundancy .In this paper, the resampling process by skipping the empty voxel is improved according to the medical image characteristics existing concentrated empty voxel. Experimental results demonstrate that the improved algorithm can accelerate the rendering speed and maintenance the quality of rendering image simultaneously.
\end{abstract}

\section{Introduction}

With the maturity and improvement of three-dimensional reconstruction technology, the medical image visualization ${ }^{[1]}$ is developing increasingly. So far, it is one of the most active areas by the researchers. This technology covers many subjects such as Computer Graphics and Image Processing Technology, and its application in medical image is aiming at processing a set of sequence of two-dimensional images for realistic 3D stereo image, then showing the human organs of 3D structure. Because the 3D image has enough information, realistic visual characteristics, this technology has great research value in clinical medicine, leading treatment and so on.

There are two types of methods in 3D reconstruction about the medical image visualization: Surface Rendering ${ }^{[2]}$ and Volume Rendering ${ }^{[3]}{ }^{[4]}$.Surface Rendering can be effective to map the surface of the object, but it cannot detect the information inside. However, the actual data inside the original image can be observed by using Volume Rendering. Ray-casting algorithm is a common algorithm of volume rendering. The basic principle of the ray-casting algorithm is a resampling and compositing process. Unfortunately, due to the large amount of calculation, the process will be in slow speed and affect the real-time rendering. In the past time, some accelerating algorithms have been put forward in order to relieve this problem, Such as volume ray-casting with differential sampling ${ }^{[5]}$, GPU-based ray casting techniques ${ }^{[6]}$.In this paper, the improved accelerating algorithm for the large amount of data to be processed and at the same time, the multi-step length will be set adaptively based on space leaping algorithm ${ }^{[7]}$. This two methods are combined effectively to reduce redundancy sampling and speed the volume rendering. Furthermore, Visualization Toolkit will be used to implement the improved algorithm and extension functions-translation, rotation, zoom, will also be implemented .

\section{Ray-Casting algorithm}

The principle of Ray-Casting algorithm. Ray-Casting algorithm, the direct volume rendering algorithm is proposed by Marc Levoy ${ }^{[8]}$.the principle is as follows: a ray is shot out from a specific point in the screen, and the ray passes through the volume data, then equidistant sampling is obtain along the ray (the model is illustrated in fig.1).the value at every sampling point is repeated accumulated according to its color value and opacity this composited voxel can be presented the every sampling point in this ray. Eventually, the synthesis value of sampling in every ray show the map image. 


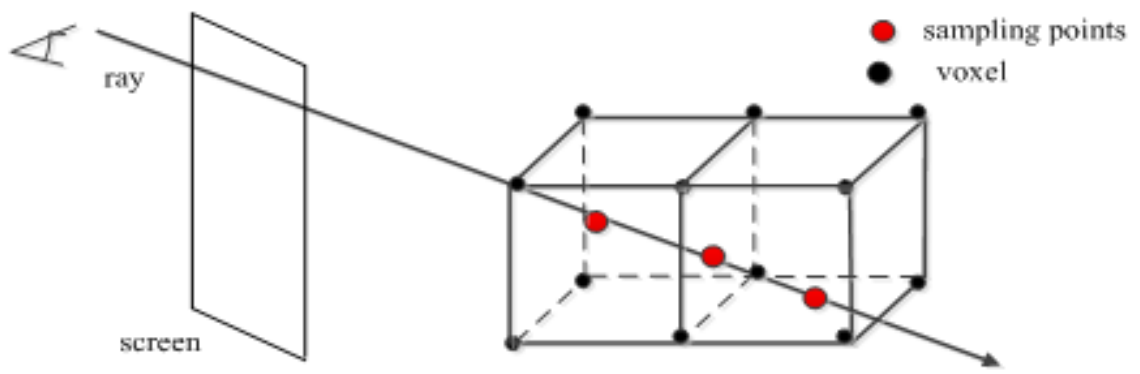

Fig. 1 The model of sampling

Empty voxel jump technique. If there exists empty voxel along the direction of the ray, all operations about these empty voxel will be in vain according to the traditional ray-casting algorithm, because it is nothing to do for the results. And exactly, in medical images, the existence of several independent organs lead to a large number of empty voxel. If to deal with all the voxel, it will inevitably increase the computational overhead, affect the image rendering speed.

In the ray-casting algorithm, there are two states for the ray:

1. To search the non-empty voxel through the empty voxel.

2. Accumulate the color and transparency about the non-empty voxel.

The first state has not any contribution for reconstruction, so these empty voxel should be skipped as far as possible to accelerate the process, and it will not affect the display result at the same time. In addition to the above mentioned by setting the step to speed up over the empty voxels, a premise of detection should also be done, To detect whether there are non-empty voxels along the ray, if not, it is marked as invalid ray that should not be made any operation, just to the next casting ray. In a word, only resample the effective volume data..

Improved Algorithm. Equidistant sampling along the radial direction is used in the traditional ray-casting algorithm. In this paper, the method of setting three-step length is introduced to change the traditional fixed step sampling method: When the particular area of given object contains a lot if empty voxels, the longer step value is set; When voxel density, the shorter step is set to increase the degree of accuracy. The purpose of this method is the same with the adaptive resampling scheme ${ }^{[9]}$, but the principle is simpler. the main idea of this improved algorithm is resampling and synthesizing on the non-empty voxels to reduce the invalid computation and improve efficiency (the process is illustrated in fig.2).

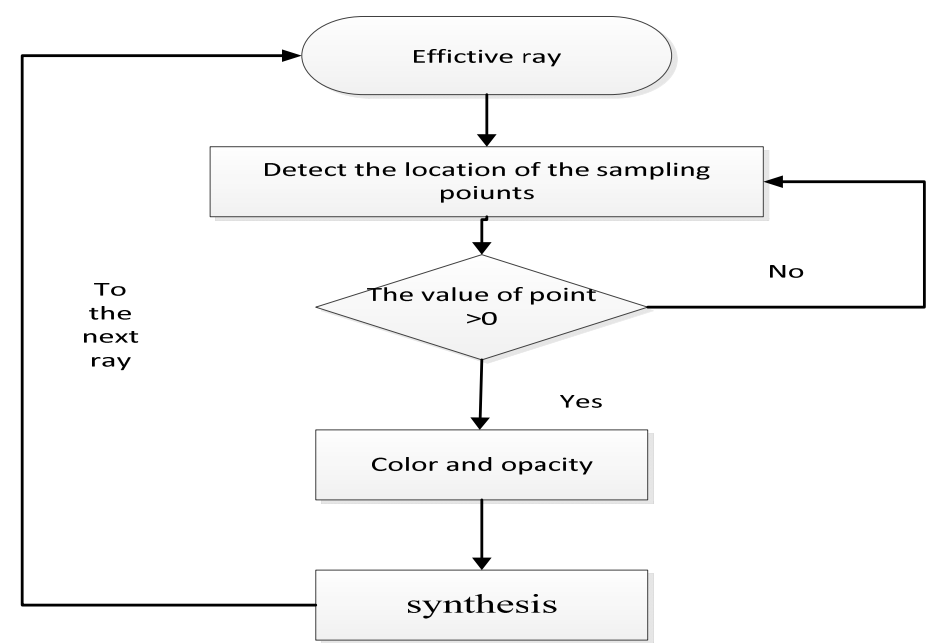

Fig. 2 Algorithm flow chart

VTK application in medical image visualization. The Visualization Toolkit (VTK) ${ }^{[10]}$ is a freely available software system for 3D computer graphics, image processing and visualization. VTK consists of a C++ class library and several interpreted interface.it is researched widely used in visualization recently. In this paper, the improved algorithm will be implemented by VTK.

In Visualization Toolkit, there are many kinds of methods about ray-casting algorithm, and the 
class named vtkVolumeRayCastingFunction is the base class as the interface for ray-casting algorithm .We can inherit the base class to encapsulate the improved algorithm.A new function class--vtkMyVolumeRayCastFunction inherited vtkVolumeRayCastingFunction is created in this test. Then we put this new class to the filter mechanism in VTK. And the medical images visualization and interaction will be completed in systematization (the process is illustrated in fig.3).

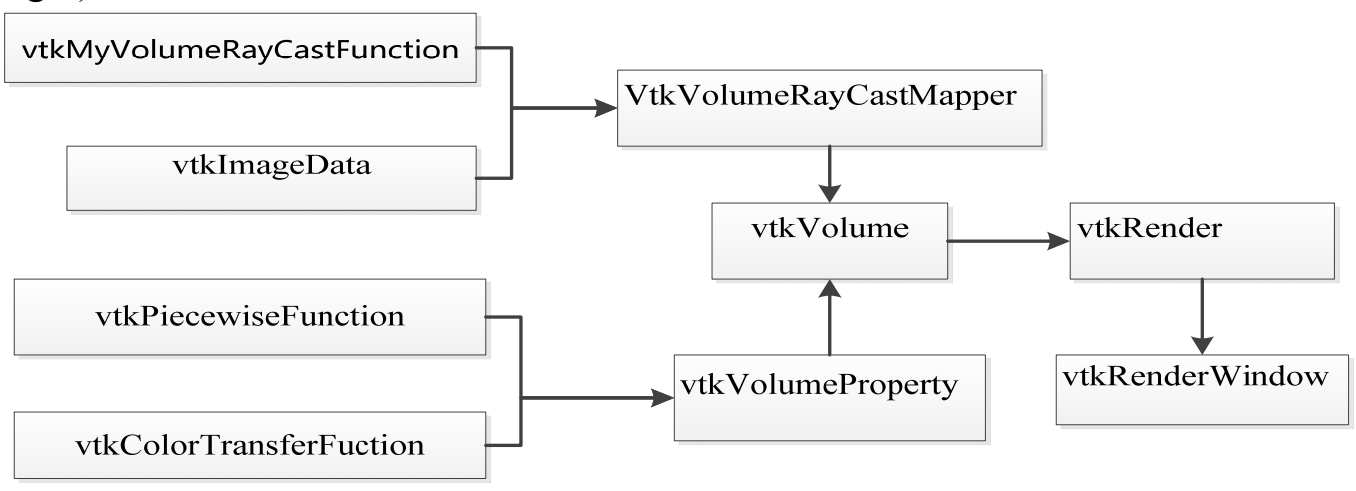

Fig 3. Volume rendering process in VTK

\section{Experimental result and analysis.}

The experimental software environment: Window 7, Visual Studio 2010, VTK5.10.1

Data source: virtual human body' head CT scan data.

When the interaction is operated to the images, the volume data will be reset to recalculation. So the time spend rotation at a time will be as the run time of the volume rendering. In the process of the rotation, there is a vague head pieces in the image (as shown in fig.4).Like the picture on the right(b), due to the empty voxels jump and the influence of uneven sampling, obvious porphyritic appear ,but it does not affect the resulting result(as shown in fig.5).

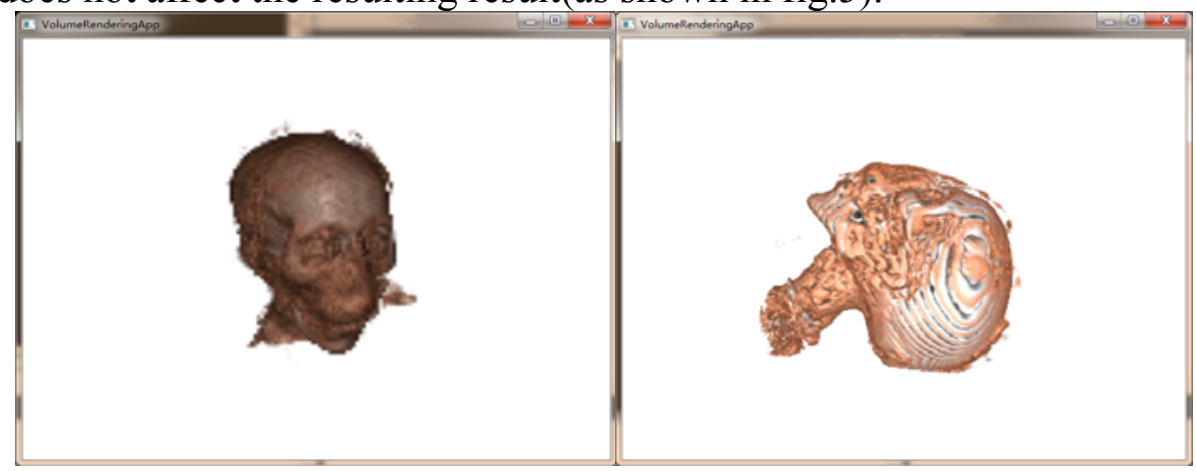

(a) The original

(b) The improved

Fig. 4 the process of volume rendering

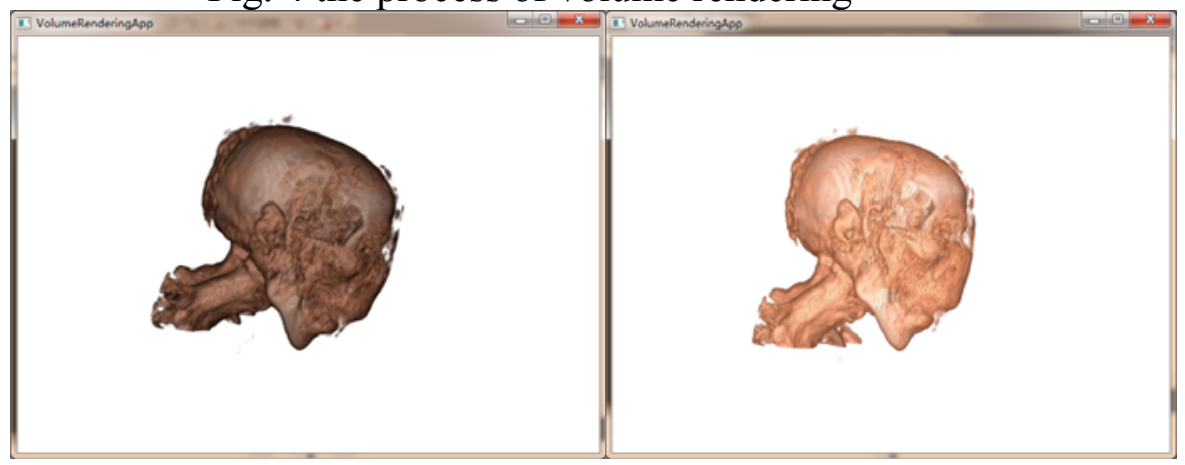

(a) The original

(b) The improved

Fig. 5 The result of volume rendering

The result of volume rendering in fig.5(b) is the almost same performance with the original(a). It 
reaches the vision under the premise of not changing 2D sequence images to speed the volume rendering and not reducing the resulting image performance. In addition, we can get the any direction image information using VTK. So that the volume rendering based on space jump empty voxel ray-casting algorithm combined with VTK is an effective way to improve medical image visualization.

\section{Conclusions}

In this paper, the empty voxel skip and the three-step jump with VTK are put forward, then through the experiments this improved algorithm achieve the goal mentioned above, the proposed methods exhibit favorable performance and it is also credible that VTK as a tool to research the 3D reconstruction has wide application space in the near future.

\section{References}

[1] Yuxuan Huang,Zhongpan Qiu,Zhijun Song. 3D reconstruction and visualization from 2D CT images[C].IEEE International Symposium on IT in Medicine and Education.2011,2:153-157.

[2] Levoy M. Display of surface from volume data[J].IEEE Computer Graphics Applications, 1988,8(3):29-37.

[3] Wenlian Cheng,chunxiao Chen,Zhiyu Qian and Lina Lu. Research On Medical Image Three Dimensional Visualization System. IEEE/ICME International Conference on Complex Medical Engineering,2007:919-923

[4] Ogata M, Ohkami T, Lauer H C, et al. A real-time volume rendering architecture using an adaptive resampling scheme for parallel and perspective projections $[\mathrm{C}] / /$

Processdings of the IEEE Symposium on Volume Visualization.1998:31-38.

[5] Knoll A , Hjazi Y, Westerteiger R, et al. Volume ray-casting with peak finding and differential sampling $[\mathrm{J}]$. IEEE Transactions on Visualization and Computer

Graphic,2009,15(6):1571-1578.

[6] Nicole S, SchollI. Comparing GPU-based multi-volume ray casting techniques[J]. Computer Science Research and Development,2011,26:39-50.

[7] Price K,Storm R.Differential Evolution--A Simple and Efficient Adaptive Scheme for Global Optimization over Continuoous Spaces[J].Journal Global Optimization over continuous Spaces[J].Journal Global Optimization,1997,11(4):341-359.

[8] Levoy M. Display of surface from volume data[J].IEEE Computer Graphics Applications, 1988,8(3):29-37.

[9] Jianguo Zhang, Zhenwei Li. Study on volume rendering of CT slices based on ray casting[C] Computer Science and Information Technology (ICCSIT), 2010 3rd IEEE International Conference on.IEEE,2010,7:157-160.

[10] William J.Schroeder.VTK User's Guide[M].Kitware.Inc,2010:108-120. 